\title{
Analysis and Comparison of Eigenspace-based Face Recognition Approaches
}

\author{
Pablo Navarrete and Javier Ruiz-del-Solar \\ Department of Electrical Engineering, Universidad de Chile, CHILE \\ Email: \{pnavarre, jruizd\}@ cec.uchile.cl
}

\begin{abstract}
Different eigenspace-based approaches have been proposed for the recognition of faces. They differ mostly in the kind of projection method been used and in the similarity matching criterion employed. The aim of this paper is to present a comparative study between some of these different approaches. This study considers theoretical aspects as well as simulations performed using a face database with a few number of classes (Yale) and a face database with a large number of classes (FERET).
\end{abstract}

Keywords: PCA, Fisher Linear Discriminant, Evolutionary Pursuit, Eigenfaces, Fisherfaces, FERET.

\section{Introduction}

Among the most successful approaches used in face recognition we can mention eigenspace-based methods, which are mostly derived from the Eigenface-algorithm [9]. These methods project the input faces onto a dimensional reduced space where the recognition is carried out, performing a holistic analysis of the faces. Different eigenspace-based approaches have been proposed. They differ mostly in the kind of projection/decomposition method been used and in the similarity matching criterion employed, which show different statistical properties. The aim of this paper is to present an independent, comparative study among some of these different approaches, focusing on the confirmation of the theoretical properties. We believe that to carry out an independent study is important, because comparisons are normally performed using the own implementations of the research groups that have proposed each method (e.g. in FERET contests), which does not consider completely equal working conditions (e.g. exactly the same preprocessing steps). Very often, more than a comparison between the capabilities of the methods, a contest between the abilities of the research groups is performed. Additionally, not all the possible implementations are considered (e.g. $p$ projection methods with $q$ similarity criteria), but only the ones that some groups have decided to use.

This comparative study considers the use of three different projection methods (Principal Component Analysis, Fisher Linear Discriminant and Evolutionary Pursuit) and five different similarity matching criteria (Euclidean-, Cosines- and Mahalanobisdistance, Self-Organizing Map and Fuzzy Feature Contrast). The pre-processing aspects of these approaches (normalization, illumination invariance, geometrical invariance, etc.) are not going to be addressed in this study. The mentioned methods are described in section 2, and the comparative study is presented in section 3 . In section 4 some conclusions are given. 


\section{Eigenspace-based Approaches}

Eigenspace-based approaches approximate the face vectors (face images) with lower dimensional feature vectors. The main supposition behind this procedure is that the face space (given by the feature vectors) has a lower dimension than the image space (given by the number of pixels in the image), and that the recognition of the faces can be performed in this reduced space. These approaches consider an off-line phase or training, where the face database is created and the projection matrix, the one that achieve the dimensional reduction, is obtained from all the database face images. In the off-line phase are also calculated the mean face and the reduced representation of each database image. These representations are the ones to be used in the recognition process.

\subsection{General Approach}

Figure 1 shows the block diagram of a generic eigenspace-based face recognition system. A preprocessing module transforms the face image into a unitary vector and then performs a subtraction of the mean face $(\overline{\mathbf{x}})$. After that, the resulting vector $\mathbf{x}$ is projected using the projection matrix $\mathbf{W} \in R^{N \times m}$ that depends on the eigenspace method been used (see section 2.2). This projection corresponds to a dimensional reduction of the input, starting with vectors $\mathbf{x}$ in $R^{N}$ (with $N$ the image vector dimension) and obtaining projected vectors $\mathbf{q}$ in $R^{m}$ with $m<N$ (usually $m<<N$ ). The Similarity Matching module compares the similarity of the reduced representation of the query face vector $\mathbf{q}$ with the reduced vectors $\mathbf{p}^{k} \in R^{m}$ that represent the faces in the database. By using a given criterion of similarity (see section 2.3), this module determines the most similar vector $\mathbf{p}^{k}$ in the database. The class of this vector is the result of the recognition process, i.e. the identity of the face. In addition, a Rejection System for unknown faces is used if the similarity matching measure is not good enough (see description in [2]).

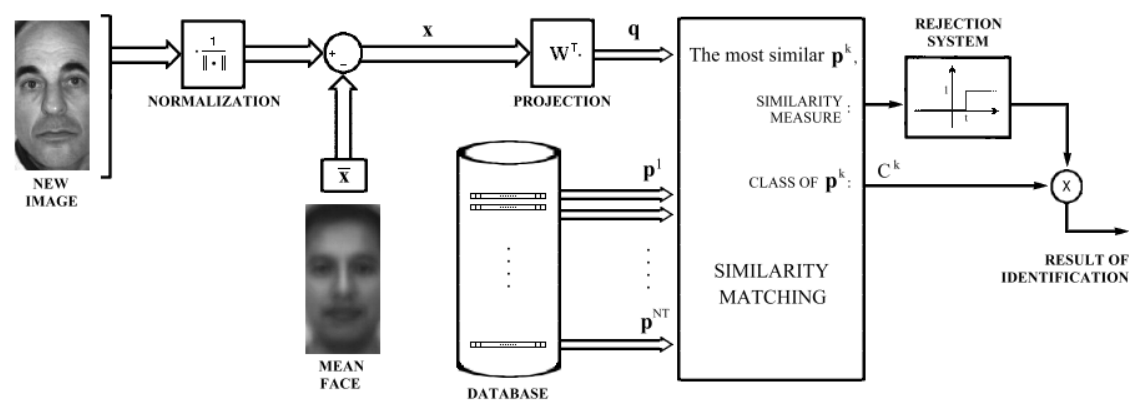

Fig. 1. Block diagram of a given eigen-space face recognition system.

\subsection{Projection/Decomposition Methods}

Principal Components Analysis - PCA 
PCA is a general method to identify the linear directions in which a set of vectors are best represented and after that to make a dimensional reduction of them. In 1987 Sirovich and Kirby [7] used Principal Component Analysis (PCA) in order to obtain a reduced representation of face images. Then, in 1991 Turk and Pentland [9] used the PCA projections as the feature vectors to solve the problem of face recognition, using the euclidean distance as the similarity function. In order to obtain the eigenfaces, we first need to obtain the projection axes in which exists the largest variance of the projected face images. Then, we repeat this procedure in the orthogonal space that is still uncovered, until we realize that there is no more variance to take into account. The theoretical solution of this problem is well known and is obtained by solving the eigensystem of the correlation matrix $\mathbf{R} \in R^{N \times N}$ :

$$
\mathbf{R}=\mathrm{E}\left\{(\mathbf{x}-\overline{\mathbf{x}})(\mathbf{x}-\overline{\mathbf{x}})^{\mathrm{t}}\right\}
$$

where $\mathbf{X}$ represent the normalized image vectors, $\overline{\mathbf{x}}$ is the mean face image, and $N$ is the original vector image dimension. The eigenvectors of this system represent the projection axes or eigenfaces, and the eigenvalues represent the projection variance of the correspondent eigenface. Then by sorting the eigenfaces in descendent order of eigenvalues we have the successive projection axes that solve our problem.

The main problem is that $\mathbf{R} \in R^{N \times N}$ is too big for a reasonable practical implementation. and then we need to The estimation of the correlation matrix is obtained, using a database with $N T$ face images (the training set), by taking the corresponding averages in the training set. Let $\mathbf{X}=\left[\left(\mathbf{x}^{1}-\overline{\mathbf{x}}\right)\left(\mathbf{x}^{2}-\overline{\mathbf{x}}\right) \cdots\left(\mathbf{x}^{N T}-\overline{\mathbf{x}}\right)\right]$ be the matrix of the normalized training vectors. Then, the $\mathbf{R}$ estimator will be given by $\mathbf{R}=\mathbf{X} \mathbf{X}^{\mathrm{T}}$. We could say that the number of eigenfaces must be less than, or equal to, $N T$, because with $N T$ training images all the variance must be projected into the hyperplane subtended by the training images. In other words the rank of $\mathbf{R}$ is less than, or equal to, NT. Thereafter they could have more null or negligible eigenvalues depending on the linear dependence of the vectors in the training set. In addition, the eigensystem of $\mathbf{X}^{\mathrm{T}} \mathbf{X} \in R^{N T \times N T}$ has the same non-zero eigenvalues of $\mathbf{R}$, because $\mathbf{X} \mathbf{X}^{\mathrm{T}} \mathbf{X} \mathbf{v}^{k}=\lambda_{k} \mathbf{X} \mathbf{v}^{k}$ represent both systems at the same time.

Now we can solve the reduced eigensystem of $\mathbf{X}^{\mathrm{T}} \mathbf{X} \in R^{N T \times N T}$. The correspondent eigenvalues are the same eigenvalues of the original system, and the eigenfaces are represented by $\mathbf{w}^{k}=\mathbf{X} \mathbf{v}^{k}$, and to be normalized they must be divided by $\sqrt{\lambda_{k}}$.

To improve the dimensional reduction, it is recommendable to neglect the components with smallest projection variances. If we ignore some components, the mean square error of the given representation is the sum of the eigenvalues not used in the representation. Therefor, a good criterion would be to choose only $m$ components, obtained by the normalized Residual Mean Square Error [8]:

$$
\operatorname{RMSE}(m)=\sum_{k=m+1}^{N} \lambda_{k} / \sum_{k=1}^{N} \lambda_{k}
$$


Considering $m$ given by $\operatorname{RMSE}(m)<5 \%$ will be good for standard applications.

\section{Fisher Linear Discriminant - FLD}

FLD searches for the projection axes on which the face images of different classes are far from each other (similar to PCA), and at the same time where the images of a same class are close from each other. In order to define the mathematical structure under FLD, first we define the parameter $\gamma(\mathbf{u})$ to be maximized on the successive projection axes as:

$$
\gamma(\mathbf{u})=\frac{s_{b}(\mathbf{u})}{s_{w}(\mathbf{u})}
$$

where $\mathbf{u}$ represents any unitary projection vector in the image space, and $s_{b}(\mathbf{u})$ and $s_{w}(\mathbf{u})$ are given by:

$$
\begin{aligned}
& s_{b}(\mathbf{u})=\sum_{i=1}^{N C} \mathrm{P}\left(C_{i}\right)\left\{\left(\mathbf{m}^{(i)}-\mathbf{m}\right) \mathbf{u}\right\}^{2}, \\
& s_{w}(\mathbf{u})=\sum_{i=1}^{N C} \mathrm{P}\left(C_{i}\right) \mathrm{E}\left[\left\{\left(\mathbf{x}^{(i)}-\mathbf{m}^{(i)}\right) \mathbf{u}\right\}^{2}\right]
\end{aligned}
$$

where $\mathbf{m}$ is the global mean vector, $\mathrm{P}\left(C_{i}\right)$ are the probabilities associated to each class $C_{i}, \mathbf{m}^{(i)}$ are the average vectors of $C_{i}$, and $\mathbf{x}^{(i)}$ are the vectors associated to $C_{i}$. In this way, $s_{b}(\mathbf{u})$ measures the separation between the individual class means respect to the global mean face, and $s_{w}(\mathbf{u})$ measure the separation between vectors of each class respect to their own class mean. Alternatively, scatter matrices are defined by:

$$
\begin{aligned}
& \mathbf{S}_{b}=\sum_{i=1}^{N C} \mathrm{P}\left(C_{i}\right)\left(\mathbf{m}^{(i)}-\mathbf{m}\right)\left(\mathbf{m}^{(i)}-\mathbf{m}\right)^{\mathrm{T}} \\
& \mathbf{S}_{w}=\sum_{i=1}^{N C} \mathrm{P}\left(C_{i}\right) \mathrm{E}\left[\left(\mathbf{x}^{(i)}-\mathbf{m}^{(i)}\right)\left(\mathbf{x}^{(i)}-\mathbf{m}^{(i)}\right)^{\mathrm{T}}\right]
\end{aligned}
$$

and then:

$$
\gamma(\mathbf{u})=\frac{\mathbf{u}^{\mathrm{T}} \mathbf{S}_{b} \mathbf{u}}{\mathbf{u}^{\mathrm{T}} \mathbf{S}_{w} \mathbf{u}} .
$$

At this point is not difficult to demonstrate that the solution of our maximization problem is the solution of the generalized eigensystem [1]:

$$
\mathbf{S}_{b} \mathbf{w}^{k}=\lambda_{k} \mathbf{S}_{w} \mathbf{w}^{k}
$$


$\mathbf{w}^{k}$ would be the fisherfaces and $\lambda_{k}$ are the successive $\gamma$ parameters associated with each fisherface. The system given by (9) is conventionally solved by writing $\mathbf{S}_{w}^{-1} \mathbf{S}_{b} \mathbf{w}^{k}=\lambda_{k} \mathbf{w}^{k}$. Notice that this eigensystem does not have orthogonal eigenvectors because $\mathbf{S}_{w}^{-1} \mathbf{S}_{b}$ is not symmetric in general, then the fisherfaces would not be an orthogonal projection set. Another implementation problems are: the matrices $\mathbf{S}_{b}$ and $\mathbf{S}_{w}$ are too big, and also $\mathbf{S}_{w}$ could be singular and then noninvertible. An easy way to solve these two problems, is to use a PCA decomposition previous to the FLD procedure. Then, the size of the scatter matrices would be small enough and depending on the criterion for dimensional reduction used, $\mathbf{S}_{w}$ will became non-singular. In this case, the eigensystem (9) will give reduced eigenvectors $\mathbf{v}^{k}$, that need to be transformed into the true eigenvectors $\mathbf{w}^{k}$ using $\mathbf{W}_{F F}=\mathbf{V} \mathbf{W}_{E F}$, where $\mathbf{W}_{E F}$ and $\mathbf{W}_{F F}$ are the PCA and Fisher projection matrices, respectively.

With two classes we need only one $\gamma$ to describe de between/within class scatter, then the projection space might have just one dimension. In the general case with $N C$ classes, we need $N C-1$ values of $\gamma$, and this corresponds to the dimension of the projection space. Given that (6) is the sum of $N C$ matrices of rank one or less, $N C$ - 1 of them are independent, and this gives $N C-1$ possible values of non-zeros $\gamma$ in (8) [1]. Thereafter we could adjust a criterion for the Fisher-space dimensional reduction. In analogy with PCA a good criterion would be to choose only $m$ components $(m \leq N C-1)$ obtained by the normalized Residual Fisher Parameter:

$$
\operatorname{RFP}(m)=\sum_{k=m+1}^{N C-1} \lambda_{k} / \sum_{k=1}^{N C-1} \lambda_{k}
$$

Considering $m$ given by $\operatorname{RFP}(m)<10^{-5} \%$ will be good for standard applications. The advantage of FLD against PCA is that the information kept in the dimensional reduction is better for recognition purposes. However, there exists some drawbacks because FLD uses the particular class information and then is recommended to have many images per class in the training process, or at least a good characterization of each one. In other words, in PCA the convergence of the $\mathbf{R}$ estimator depends mostly on the total number of target images $N T$, but in FLD the convergence of the scatter matrices estimators depends also on the numbers of target images per class. Then, the main drawback of FLD is that it could be over-adjusted on the target images, and then the recognition system may have an important lack of generalization that may be notice in the resulting system's recognition rate.

\section{Evolutionary Pursuit - EP}

The eigenspace-based adaptive approach proposed in [4], searches for the best set of projection axes in order to maximize a fitness function, measuring at the same time the classification accuracy and generalization ability of the system. Because the dimension of the solution-space of this problem is too large, it is solved using a 
specific kind of Genetic Algorithm called Evolutionary Pursuit (EP). In the proposed representation, we are going to call the projection axes (image vectors) as EP-faces.

In order to obtain the EP-faces first is done an initial dimensional reduction using PCA, and then is used the Whitening Transformation (equivalent to a Mahalanobis metric system, see 2.3). In the Whitened-PCA space several rotations between pair of axes are applied and a subset of the rotated Whithened-PCA axes is selected. This transformation is coded using a chromosome representation. The chromosome structure use 10 bit for each angle $\alpha_{k}$ (between 0 and $\pi / 2$ ), and a number of bits $a_{i}$ equal to the number of Whithened-PCA components $(m)$ in order to select a subset of axes. Notice that the number of possible rotations between axes would be $m(m-1) / 2$, so the number of bits for each chromosome is $5 m(m-1)+m$, and the size of the genome-space (too large to search it exhaustively) is $2^{5 m(m-1)+m}$.

Each chromosome represents a certain projection system. Then in order to evaluate this system the following fitness function is used:

$$
\zeta\left(\alpha_{k}, a_{i}\right)=\zeta_{a}\left(\alpha_{k}, a_{i}\right)+\lambda \zeta_{s}\left(\alpha_{k}, a_{i}\right) .
$$

$\zeta_{a}\left(\alpha_{k}, a_{i}\right)$ measures the accuracy, $\zeta_{s}\left(\alpha_{k}, a_{i}\right)$ measures the generalization ability, and $\lambda$ is a positive constant that determines the importance of the second term against the first one. The generalization ability is computed as (remember $\mathbf{S}_{b}$ given by (6)):

$$
\zeta_{s}\left(\alpha_{k}, a_{i}\right)=\sqrt{\sum_{\mathrm{i}=1}^{\mathrm{NC}}\left(\mathbf{m}^{(i)}-\mathbf{m}\right)^{\mathrm{T}}\left(\mathbf{m}^{(i)}-\mathbf{m}\right)} .
$$

where $\mathbf{m}$ is the global mean and $\mathbf{m}^{(i)}$ is the mean of the corresponding class $C_{i}$. Although the accuracy measure $\zeta_{a}\left(\alpha_{k}, a_{i}\right)$ proposed in [4] is the recognition rate of training face images as the top choice, in our implementation we used the top 2 identity because with the top choice it was too easy to obtain a $100 \%$ recognition rate.

As usual, in order to find the maximal value of the fitness function, a random set of chromosomes is randomly set and the GA procedure iterates until the system converges. The operators used to create a new set of chromosomes per iteration are: proportionate selection, pre-selection of parents in proportion to their relative fitness; two-point crossover, exchange the selection between the crossover points; and fixed probability mutation, each bit of the chromosome is given a fixed probability of flipping. Details of this procedure can be found in [4].

\subsection{Similarity Matching Methods}

\section{Euclidean Distance}

$$
d(\mathbf{x}, \mathbf{y})=\sqrt{(\mathbf{x}-\mathbf{y})^{\mathrm{T}}(\mathbf{x}-\mathbf{y})} .
$$

\section{Cosine Distance}




$$
\cos (\mathbf{x}, \mathbf{y})=\frac{\mathbf{x}^{\mathrm{T}} \mathbf{y}}{\|\mathbf{x}\|\|\mathbf{y}\|}
$$

\section{Mahalanobis Distance}

$$
d(\mathbf{x}, \mathbf{y})=(\mathbf{x}-\mathbf{y})^{\mathrm{T}} \mathbf{R}^{-1}(\mathbf{x}-\mathbf{y}) ; \quad \mathbf{R} \text { : correlation matrix. }
$$

From a geometrical point of view this distance has a scaling effect in the image space. Taking into consideration the face image subset, directions in which a greater variance exist are compressed and directions in which a smaller variance exist are expanded. It can be proved that in the PCA space the Mahalanobis distance is equivalent to the Euclidean distance, weighting each component by the inverse correspondent eigenvalue (see demonstration in [5]), and it is often called Whitening (PCA) Transformation.

\section{SOM Clustering}

Self-Organizing Maps (SOM) [3] are used as associative networks to match the projected query face with the corresponding projected database face. The use of a SOM to implement this module could improve the generalization ability of the system. The SOM approach uses reference vectors $\mathbf{m}_{i}$, the so-called SOM codewords, to approximate the probability distribution of the faces in a 2D map. In the training phase of the SOM a clustering of the reduced vector faces is carried out. Thereafter the SOM is transformed in an associative network by a labeling of all his nodes. To find the reference vectors, the following step is to find the best matching of each training image and to see in which place of the map they are going to be located. After that a so-called labeling algorithm allows to associate a fixed face identity for each node in the map.

\section{Fuzzy Feature Contrast - FFC}

$$
\begin{aligned}
S(\mathbf{x}, \mathbf{y})=\sum_{i=1}^{m} \min \left\{\mu_{i}(\mathbf{x}), \mu_{i}(\mathbf{y})\right\} & -\alpha \sum_{i=1}^{m} \max \left\{\mu_{i}(\mathbf{x})-\mu_{i}(\mathbf{y}), 0\right\} \\
& -\beta \sum_{i=1}^{m} \max \left\{\mu_{i}(\mathbf{y})-\mu_{i}(\mathbf{x}), 0\right\}
\end{aligned}
$$

where $\mu_{i}(\mathbf{x})$ is a membership function associated with the i-component of vector $\mathbf{x} \in R^{m}$.

This similarity measure, originally proposed in [6], is a fuzzy implementation of the Feature Contrast model from Tversky. The first sum measure the common features (intersection) and the others represent the distinctive features (difference in the two possible ways). The positive parameters $\alpha$ and $\beta$ adjust the contrast of the three kind of features. By choosing $\alpha \neq \beta$ it is possible to introduce asymmetries between the subject-referent comparison. This model considers that all the features are independent, and that can be assumed in PCA and WPCA, but not in FLD and EP. 
In our implementation we normalize each feature of PCA (in WPCA it is not necessary) and we chose $\mu_{i}(\mathbf{x})$ linear between -1 and 1 , with $x_{i}$ normalized.

\section{Comparison among the approaches}

\subsection{Simulations using the Yale Face Image Database}

In order to compare the described methods we have first made several simulations using the Yale University - Face Image Database [11]. We used 150 images of 15 different classes. First, we preprocessed the images manually by masking them in windows of $100 \times 200$ pixels and centering the eyes in the same relative places. In table 1 we show the results of several simulations using different kind of representations and similarity matching methods. For each simulation we used a fixed number of training images, using the same type of images per class, according with the Yale database specification. In order to obtain representative results we take the average of 20 different sets of images for each fixed number of training images. All the images not used for training are used for testing.

We can see that the best results are always obtained with the FLD representation, and the difference against other representations decrease when the number of training images per class decrease, showing that the FLD discrimination ability strongly depends on the number of training images per class. The best results are almost always obtained with FLD- cosine. The systems that seem to be as efficient as FLDcosine are FLD-SOM and FLD-Withening-cosine.

The best results using FFC were obtained employing an asymmetric subjectreferent comparison: $\alpha=0.5 \beta=5$. This means that in the question "how is the subject face similar to the referent face?" the answer focus more on the features of the referent (the unknown face). The generalization ability of the systems is not well measured in these simulations because the number of selected axes is about the same as the number of classes (15). That affects the EP representation method as well as the FFC and SOM similarity matching methods (see section 4.3).

\subsection{Simulations using FERET}

In order to test the described methods using a large database, we made several simulations using FERET database [10]. We use a target set with 762 images of 254 different classes ( 3 images per class), and a query set of 254 images ( 1 image per class). Eyes location is included in FERET database for all the images being used. Then the preprocessing consists in centering and scaling images so that eyes position keep in the same relative place. In table 2 we show the results of simulations using different kind of representations and similarity matching methods. In this table the SOM similarity measure was not included because in these tests the number of classes (254) is much larger than the number of images per class ( 2 or 3 ), and the training process is very difficult. It is very unlikely that new images are well classified in a SOM net in which too little nodes were labeled with the correct identity. 
Table 1. Mean recognition rates using different numbers of training images per class, and taking the average of 20 different training sets. The small numbers are the standard deviation of each recognition rate. All results consider the top 1 match for recognition. Yale Database.

\begin{tabular}{|c|c|c|c|c|c|c|c|c|c|c|}
\hline $\begin{array}{c}\text { projection } \\
\text { method }\end{array}$ & $\begin{array}{l}\text { images } \\
\text { per class }\end{array}$ & axes & euclidean & $\cos (\cdot)$ & SOM & FFC & $\begin{array}{l}\text { whitening } \\
\text { euclidean }\end{array}$ & $\begin{array}{c}\text { whitening } \\
\cos (\cdot)\end{array}$ & $\begin{array}{c}\text { whitening } \\
\text { SOM }\end{array}$ & $\begin{array}{c}\text { whitening } \\
\text { FFC }\end{array}$ \\
\hline PCA & & 56 & $\begin{array}{c}87.9 \\
6.2\end{array}$ & $\begin{array}{c}86.0 \\
6.8\end{array}$ & $\begin{array}{c}84.6 \\
7.0\end{array}$ & $\begin{array}{c}77.1 \\
10.1\end{array}$ & $\begin{array}{c}64.7 \\
9.4\end{array}$ & $\begin{array}{c}79.3 \\
11.6\end{array}$ & $\begin{array}{c}64.7 \\
10.5\end{array}$ & $\begin{array}{c}77.1 \\
10.1\end{array}$ \\
\hline FLD & 6 & 14 & $\begin{array}{c}91.5 \\
6.6\end{array}$ & $\underset{6.5}{91.6}$ & $\begin{array}{c}90.3 \\
6.7\end{array}$ & $\begin{array}{c}83.9 \\
9.3\end{array}$ & $\begin{array}{c}91.9 \\
5.8\end{array}$ & $\begin{array}{c}92.6 \\
5.6\end{array}$ & $\begin{array}{r}92.1 \\
6.2\end{array}$ & $\begin{array}{c}85.6 \\
8.3\end{array}$ \\
\hline E.P. & & 15 & $\begin{array}{c}81.2 \\
9.0 \\
\end{array}$ & $\begin{array}{c}85.3 \\
8.7 \\
\end{array}$ & $\begin{array}{c}83.7 \\
9.8 \\
\end{array}$ & $\begin{array}{c}77.2 \\
8.0 \\
\end{array}$ & - & - & - & - \\
\hline PCA & & 34 & $\begin{array}{c}88.7 \\
3.8\end{array}$ & $\begin{array}{c}87.1 \\
4.1\end{array}$ & $\begin{array}{c}86.0 \\
5.1\end{array}$ & $\begin{array}{c}78.5 \\
8.1\end{array}$ & $\begin{array}{c}69.5 \\
8.9\end{array}$ & $\begin{array}{c}83.2 \\
9.0\end{array}$ & $\begin{array}{c}66.1 \\
10.5\end{array}$ & $\begin{array}{c}78.5 \\
8.1\end{array}$ \\
\hline FLD & 5 & 14 & $\begin{array}{c}92.2 \\
5.7\end{array}$ & $\begin{array}{c}91.7 \\
6.2\end{array}$ & $\begin{array}{c}90.3 \\
6.4\end{array}$ & $\begin{array}{c}85.1 \\
9.1\end{array}$ & $\begin{array}{c}92.3 \\
4.7\end{array}$ & $\begin{array}{c}92.4 \\
5.7\end{array}$ & $\begin{array}{c}92.1 \\
5.3\end{array}$ & $\begin{array}{c}85.4 \\
8.5\end{array}$ \\
\hline E.P. & & 13 & $\begin{array}{c}84.1 \\
5.7 \\
\end{array}$ & $\begin{array}{c}87.7 \\
6.6 \\
\end{array}$ & $\begin{array}{c}86.7 \\
7.6 \\
\end{array}$ & $\begin{array}{c}78.7 \\
6.8 \\
\end{array}$ & - & - & - & - \\
\hline PCA & & 46 & $\begin{array}{c}87.3 \\
3.9\end{array}$ & $\begin{array}{c}86.7 \\
3.9\end{array}$ & $\begin{array}{c}84.8 \\
3.6\end{array}$ & $\begin{array}{c}77.6 \\
5.2\end{array}$ & $\begin{array}{c}72.9 \\
5.5\end{array}$ & $\begin{array}{c}84.4 \\
5.6\end{array}$ & $\begin{array}{c}66.7 \\
6.5\end{array}$ & $\begin{array}{c}77.6 \\
5.2\end{array}$ \\
\hline FLD & 4 & 14 & $\begin{array}{c}90.3 \\
4.5\end{array}$ & $\underset{5.0}{91.1}$ & $\underset{4.4}{90.3}$ & $\begin{array}{c}84.4 \\
5.9\end{array}$ & $\begin{array}{c}90.4 \\
4.2\end{array}$ & $\begin{array}{c}91.0 \\
4.4\end{array}$ & $\begin{array}{c}90.1 \\
4.7\end{array}$ & $\begin{array}{c}82.9 \\
5.7\end{array}$ \\
\hline E.P. & & 18 & $\begin{array}{r}83.6 \\
4.6 \\
\end{array}$ & $\begin{array}{c}86.9 \\
4.7 \\
\end{array}$ & $\begin{array}{c}85.0 \\
5.0 \\
\end{array}$ & $\begin{array}{r}74.7 \\
6.0 \\
\end{array}$ & - & - & - & - \\
\hline PCA & & 35 & $\begin{array}{c}86.6 \\
4.0\end{array}$ & $\begin{array}{c}85.4 \\
3.9\end{array}$ & $\begin{array}{c}82.0 \\
5.6\end{array}$ & $\begin{array}{c}77.9 \\
4.6\end{array}$ & $\begin{array}{c}75.0 \\
5.6\end{array}$ & $\begin{array}{c}84.8 \\
5.4\end{array}$ & $\begin{array}{c}67.4 \\
6.9\end{array}$ & $\begin{array}{c}77.9 \\
4.6\end{array}$ \\
\hline FLD & 3 & 14 & $\begin{array}{c}89.0 \\
3.6\end{array}$ & $\begin{array}{c}90.4 \\
4.0\end{array}$ & $\begin{array}{c}87.4 \\
4.0\end{array}$ & $\begin{array}{c}80.7 \\
6.3\end{array}$ & $\begin{array}{c}88.9 \\
3.1\end{array}$ & $\begin{array}{c}89.9 \\
3.9\end{array}$ & $\begin{array}{c}88.7 \\
3.9\end{array}$ & $\begin{array}{c}81.5 \\
3.4\end{array}$ \\
\hline E.P. & & 14 & $\begin{array}{c}81.1 \\
4.3 \\
\end{array}$ & $\begin{array}{c}86.9 \\
3.7 \\
\end{array}$ & $\begin{array}{c}82.5 \\
3.7 \\
\end{array}$ & $\begin{array}{r}75.9 \\
4.4 \\
\end{array}$ & - & - & - & - \\
\hline PCA & & 26 & $\begin{array}{c}82.7 \\
5.9\end{array}$ & $\begin{array}{c}80.8 \\
5.9\end{array}$ & $\begin{array}{c}76.2 \\
7.9\end{array}$ & $\begin{array}{c}71.1 \\
5.9\end{array}$ & $\begin{array}{c}75.6 \\
4.9\end{array}$ & $\begin{array}{c}82.1 \\
4.6\end{array}$ & $\begin{array}{c}60.8 \\
7.3\end{array}$ & $\begin{array}{c}71.1 \\
5.9\end{array}$ \\
\hline FLD & 2 & 14 & $\begin{array}{c}81.5 \\
5.6\end{array}$ & $\begin{array}{c}82.2 \\
5.8\end{array}$ & $\begin{array}{c}79.4 \\
5.8\end{array}$ & $\begin{array}{c}69.3 \\
8.6\end{array}$ & $\begin{array}{c}80.7 \\
4.7\end{array}$ & $\begin{array}{c}82.8 \\
4.9\end{array}$ & $\begin{array}{c}78.8 \\
5.8\end{array}$ & $\begin{array}{c}73.6 \\
6.2\end{array}$ \\
\hline E.P. & & 14 & $\begin{array}{c}77.8 \\
5.6\end{array}$ & $\begin{array}{c}81.2 \\
5.3\end{array}$ & $\begin{array}{c}76.0 \\
7.3\end{array}$ & $\begin{array}{c}70.0 \\
7.4\end{array}$ & - & - & - & \\
\hline
\end{tabular}

Table 2. Mean recognition rates for standard approaches using FERET database images with 3 images per class in the target set. All results consider the top 1 match for recognition.

\begin{tabular}{c|c|c|cccccc}
\hline $\begin{array}{c}\text { projection } \\
\text { method }\end{array}$ & $\begin{array}{c}\text { images } \\
\text { per class }\end{array}$ & axes & euclidean & $\cos (\cdot)$ & FFC & $\begin{array}{c}\text { whitening } \\
\text { euclidean }\end{array}$ & $\begin{array}{c}\text { whitening } \\
\cos (\cdot)\end{array}$ & $\begin{array}{c}\text { whitening } \\
\text { FFC }\end{array}$ \\
\hline PCA & & 212 & 87.0 & 88.6 & 85.0 & 74.4 & 89.4 & 85.0 \\
FLD & $\mathbf{3}$ & 108 & 91.3 & 94.1 & 92.1 & 85.8 & 92.1 & 92.1 \\
E.P. & & 115 & 91.0 & 93.1 & 91.0 & - & - & - \\
\hline PCA & & 180 & 81.9 & 83.7 & 80.7 & 62.3 & 86.0 & 80.7 \\
FLD & \multirow{2}{*}{73} & 79.5 & 88.2 & 85.2 & 79.5 & 88.2 & 85.2 \\
E.P. & & 96 & 80.3 & 85.8 & 83.3 & - & - & - \\
\hline
\end{tabular}

We can see that the best simulation results are obtained with the FLD representation, which is similar to the case when a small database is used (see section 
4.2). One of the main differences regarding the case of small databases are the results obtained using FFC similarity measure that considerably increases its recognition rate. The best results for FFC similarity measure were obtained employing an asymmetric subject-referent comparison: $\alpha=1 \beta=2$ (in this way the answer still focus more on the features of the referent). This confirms our previous hyphotesis that large number of classes will force generalization ability on this system. Also we could see that FLDcosine works better than other systems, showing us that the FLD dimensional reduction works better than other projection methods. We can also see that the EPsystems always performed worse than the FLD systems. At the same time, we realized that the number of axes selected are always of the same order of the number of classes, then the accuracy pursuit (mostly dependent on the top 2 match) seems to fail for this reason, and then FLD kept the advantage. The worst results seems to be the obtained with Whitening-PCA-Euclidean and Whitening-PCA-SOM, and against the result of the cosine-based systems we can see that the changes in the norms of the vectors seems to confuse the recognition ability.

\subsection{Computational Complexity}

Another important issue to compare the different projection methods is the computational cost of the training processes. In PCA this computational cost is mainly due to the process of determining $\mathbf{R}, O\left(N T^{2} \cdot N\right)$, and solving the eigensystem, $O\left(N T^{3}\right)$. If we suppose that the number of training images $N T$ is much smaller than the number of pixels per image $N$, then the computational cost of PCA is just the cost of determining $\mathbf{R}, O\left(N T^{2} \cdot N\right)$. In our implementation of FLD we requires previously the computation of PCA to reduce the vectors dimension to $m_{1}\left(m_{1}<N T\right)$, and the additional cost is due to the process of determining the scatter matrices, $O\left(m_{1}^{2} \cdot N C\right)$, and solving the general eigensystem, $O\left(m_{1}^{3}\right)$. Nevertheless the additional cost in FLD is usually much smaller than the PCA initial cost, $O\left(N T^{2} \cdot N\right)$. Finally EP requires much more computations because this process must iterate until a given criterion is accomplished. The computational complexity of EP strongly depends on the implementation but it usually takes more than 10 times the time of the PCA procedure.

The computational cost of on-line operation is mainly given by the comparisons with database vectors, $O(N T \cdot m)$, except when the SOM-based similarity measure is used, $O($ (number of nodes $) \cdot m$ ). The numerical stability for the different methods depends mostly of the numerical algorithms used for solving eigensystems. Either in PCA or FLD this is not a critical problem because always involves the management of symmetric matrices.

\section{Conclusions}

Eigenspace-based approaches have shown to be efficient in order to deal with the problem of face recognition. Although these methods have proved their goodness in several works, we believe that there is a necessity of a general evaluation to 
understand their differences and similarities. In this sense our goal was to provide an unified vision over the most thriving eigenspace approaches.

We made an extensive analysis of different eigenspace-based approaches, separating the representation problem from the similarity matching method employed, and using databases with small and large number of classes. We also used the Whitening Transformation as a Mahalanobis metric system before the initial PCA processing, in order to match PCA and FLD against EP. In order to obtain the best recognition rates we saw that the FLD projection method and the cosine similarity matching work better, and that the whitening-cosine based methods seem to keep their recognition ability with fewer target images. Even if the EP projection method and the FFC similarity measure did not show the best recognition abilities, they showed a good performance and a good generalization ability in the tests using FERET database, in which the other systems could not overcome the adjustment of few projection axes (in relation to the number of classes). An important aspect of this work was to change the standard block of similarity matching for a SOM. In this way the structure of the identification system (see figure 1) changes because we do not need the database reduced representation anymore. Now this information would be appropriately included in the SOM reference vectors (codewords). In our simulations we realize that this kind of identification system works as good as the standard ones, concerning the recognition rates with a small database. An interesting feature of this approach is the possibility to adapt itself to changes in the faces. This has a direct application in adaptive security access systems where the persons to be recognized would be constantly viewed by the system. Specifically, when a person arrive the neural system will carried out the recognition, and after that the SOM will perform one training iteration and the labeling procedure will be re-applied. In this way the SOM map will adapt itself to changes in the features of faces like those due to the bear, hair, or even age evolution. In this sense this system represents a robust and adaptive control access identification system.

As future work we are focusing in the extension of this study, considering another kind of eigenspace-based approaches. In this way we are interested in exploring the so-called differential approaches, that allow the application of statistical analysis in the recognition process by working with differences between face images, rather than face images.

\section{Acknowledgements}

This research was supported by FONDECYT (Chile).

Portions of the research in this paper use the FERET database of facial images collected under the FERET program.

\section{References}

1. Duda R.O., Hart P.E., and Stork D.G., "Pattern Classification”, Chap. 3, 2001.

2. Golfarelli M., Maio D., and Maltoni D., "On the Error-Reject Trade-Off in Biometric Verification Systems", IEEE Trans. Pattern Analysis and Machine Intelligence, vol. 19, no. 7, pp. 786-796, July 1997.

3. Kohonen T., "Self-Organized Maps", 1997. 
4. Liu C., and Wechsler H., "Evolutionary Pursuit and Its Application to Face Recognition", IEEE Trans. Pattern Analysis and Machine Intelligence, vol. 22, no. 6, pp. 570-582, June 2000.

5. Navarrete P., and Ruiz del Solar J., "Eigenspace-based Recognition of Faces: Comparisons and a new Approach", Proc. of the Int. Conf. on Image Analysis and Processing ICIAP 2001, pp. 42-47, Sept. 26-28, Palermo, Italy.

6. Santini S., and Jain R., "Similarity Measures", IEEE Trans. Pattern Analysis and Machine Intelligence, vol. 21, no. 9, pp. 871-883, September 1999.

7. Sirovich L., and Kirby M., "A low-dimensional procedure for the characterization of human faces", J. Opt. Soc. Amer. A, vol. 4, no. 3, pp. 519-524, 1987.

8. Swets D.L. and Weng J.J., "Using Discriminant Eigenfeatures for Image Retrieval", IEEE Trans. Patt. Analysis and Machine Intell., vol. 18, no. 8, 831-836, 1996.

9. Turk M., and Pentland A., "Eigenfaces for Recognition", J. Cognitive Neuroscience, vol. 3, no. 1, pp. 71-86, 1991.

10. Phillips P. J., Wechsler H., Huang J., and Rauss P., "The FERET database and evaluation procedure for face recognition algorithms," Image and Vision Computing J., Vol. 16, no. 5, 295-306, 1998.

11. Yale University Face Image Database, publically available for non-commercial use, http://cvc.yale.edu/projects/yalefaces/yalefaces.html 